Elsevier

HRR 00889

\title{
Cryoprobe-induced apical lesions in the chinchilla. I. Morphological effects of lesioning parameters
}

\author{
J. Nadine Brown and Alfred L. Nuttall \\ Kresge Hearing Research Institute, University of Michigan Medical School, Ann Arbor, U.S.A.
}

(Received 16 June 1986; accepted 22 November 1986)

\begin{abstract}
To create experimental lesions localized to the low frequency region of the organ of Corti, a cryoprobe was applied to the apical area of 37 cochleas from 26 adult chinchillas. Twenty cochleas were exposed to single applications of a cryoprobe for $2.5,3.0$ and $3.5 \mathrm{~min} ; 17$ cochleas were exposed to two applications of 1.5, 2.0 and $3.0 \mathrm{~min}$ each with about a 3 min interval between applications. Cryoprobe tip temperature rose from about $-140^{\circ} \mathrm{C}$ when placed on the apex to about $-80^{\circ} \mathrm{C}$ after a continuous 3.5 min application. Survival time after lesioning was from 2 to 75 days, with most being 12 days or less. All cochleas except one sustained regions of damage characterized by complete absence of the organ of Corti and by missing hair cells indicated by extensive scarring. Inner hair cells were less susceptible to damage than were outer hair cells. Well-defined lesions which were continuous over the apical organ of Corti were found in some cochleas exposed to single probe applications, but such applications more often resulted in lesions which had areas of less damage. Of the various application protocols used, two applications of 1.5 min each, with an interval to allow the tissue to warm, most consistently produced severe and discrete apical lesions. In 9 of 13 cochleas exposed to two 1.5 min probe applications, such lesions extended about $35 \%$ or less of the distance from the apex. In most cochleas, regardless of the severity of the apical lesion, the pattern of hair cell rows and stereocilia configuration appeared normal in the basal $40-50 \%$ of the organ of Corti.
\end{abstract}

Cryolesion; Apical lesion; Cryoprobe; Cochlea; Chinchilla

\section{Introduction}

Experimentally induced lesions of the cochlea are among the most valuable methods available to study auditory function. Noise and/or drugs have a long history for the creation of damage in the mid- and basal-turn of the organ of Corti. The resulting functional deficits have correlated with the observed morphological changes revealing much knowledge about encoding of acoustic information.

The loss of cochlear hair cells only in the apical region is relatively rare. Consequently, not as much opportunity has been afforded to study the contribution of the apical hair cell population to the encoding of low frequency information. For exam-

Correspondence to: A.L. Nuttall, Kresge Hearing Research Institute, University of Michigan Medical School, Ann Arbor, MI 48109, U.S.A. ple, it is not known to what extent basal turn (high frequency) hair cells may participate in reception of moderate- and high-intensity level low frequency sounds.

Although many different methods produce lesions of the cochlea [e.g., acoustic overstimulation (Engström and Ades, 1960), ototoxic drugs (Ylikoski, 1974), x-ray irradiation (Winther, 1970), ultrasound (Lundquist et al., 1978), and laser irradiation (Stahle et al., 1972)], the literature indicates that discrete damage to the apex can most easily and inexpensively be accomplished by surgical means with the application of a cryoprobe.

Effects of exposure to cold in producing experimental lesions were studied by Lundquist and associates (1972) and by Morrison and Lundquist $(1974 a, b)$ in the vestibular system of the guinea pig, and the possibility of selective destruction of tissue was noted. The investigation was extended to cochlear structures in the guinea pig by Kuylenstierna and Lundquist $(1982,1983)$ where 
morphological changes occurring after various controlled low temperatures were described. In determining the cochlea's sensitivity to cold, they produced well-defined lesions in the organ of Corti with a moderate degree of reproducibility.

A commercial cryosurgical unit was used in all of the above investigations. A simple passive cryoprobe instrument was developed by Suzuki and Hunter-Duvar (1982) in studying cryosurgical effects on sensory structures in the chinchilla cochlea. Resulting morphological damage was studied by scanning electron microscopy and was determined to be similar in many ways to that caused by loud sound or drug exposure.

The above studies suggest that cryotechniques could be developed to produce discrete apical lesions of the cochlea. The current study investigates some of the parameters necessary to produce, with reasonable consistency, cryosurgical lesions characterized by complete absence of apical hair cells.

\section{Materials and Methods}

Twenty-six adult chinchillas obtained from local breeders were subjects for this study. The animals, weighing $400-600 \mathrm{~g}$, were anesthetized with intramuscular injections of xylazine (2.5 $\mathrm{mg} / \mathrm{kg}$ ) and ketamine hydrochloride $(15 \mathrm{mg} / \mathrm{kg})$, following a subcutaneous injection of atropine $(0.04 \mathrm{mg} / \mathrm{kg})$. Under sterile conditions, the bulla was approached ventrally and an opening sufficiently large to view the apical two turns of the cochlea was made with a dental drill. The tip of a cryoprobe was placed on the apical turn for total periods ranging from 2.5 to $6.0 \mathrm{~min}$. The wound was closed and recovery was usually uneventful.

The cryoprobe designed for this procedure (Fig. 1) consists of a $35 \mathrm{~g}$ solid copper cylinder attached to a teflon handle. The cylinder serves as a heat sink and a removable $2 \mathrm{~cm} \times 1.5 \mathrm{~mm}$ copper rod at its end as the probe which touches the bony wall of the cochlea. The tip of the probe is beveled to provide increased contact surface. The entire copper portion is immersed in liquid nitrogen $\left(-196^{\circ} \mathrm{C}\right)$ for $2-3 \mathrm{~min}$ until it equilibrates in temperature. The cryoprobe tip is then immediately applied to the selected site on the apex. Cryoprobe temperature, as measured with a ther-

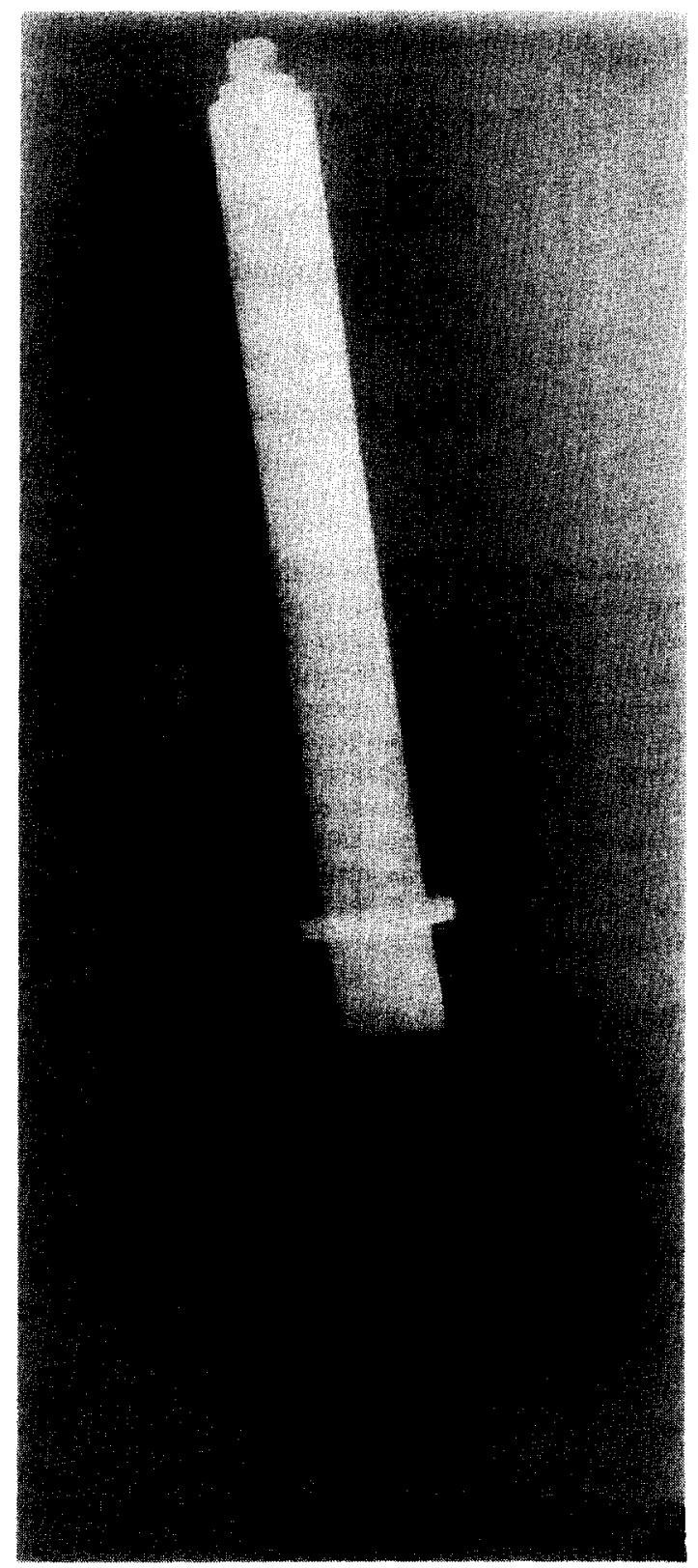

Fig. 1. The cryoprobe consists of a $35 \mathrm{~g}$ solid copper cylinder attached to a teflon handle. A removable $2 \mathrm{~cm} \times 1.5 \mathrm{~mm}$ copper rod at the end of the cylinder serves as the application tip which touches the bony wall of the cochlea.

mocouple embedded within the copper rod $1 \mathrm{~mm}$ from the tip, rose from about $-140^{\circ} \mathrm{C}$ when placed on the cochlea to about $-75^{\circ} \mathrm{C}$ when removed after a continuous $4 \mathrm{~min}$ application. 
The temperature recovery function is shown in Fig. 2.

Desired characteristics for these experimental apical lesions include (1) loss of all inner hair cells (IHCs) and outer hair cells (OHCs) for not more than $30-40 \%$ of the distance from the apex (corresponding to estimated basilar membrane frequency tuning below $1 \mathrm{kHz}$ (Eldredge et al., 1981), and (2) normal-looking sensory cells basal to the damaged area.

After initial experiments with single application durations as short as $30 \mathrm{~s}$ and as long as $4 \mathrm{~min}$, it was determined that for the protocol of a single continuous cryoprobe application, 2.5-3.5 min usually produced the most acceptable lesion, and 20 cochleas from 15 animals having this duration of exposure were studied in detail. Survival time after lesioning for these animals was from 2 to 24 days.

In experiments on subsequent animals, it was determined that multiple probe applications resulted in more reliable production of the desired lesions. In 17 cochleas from 11 animals, two cryoprobe applications of $1.5,2$ or $3 \mathrm{~min}$ each were used, approximately to the same apical site. The interval between applications was usually

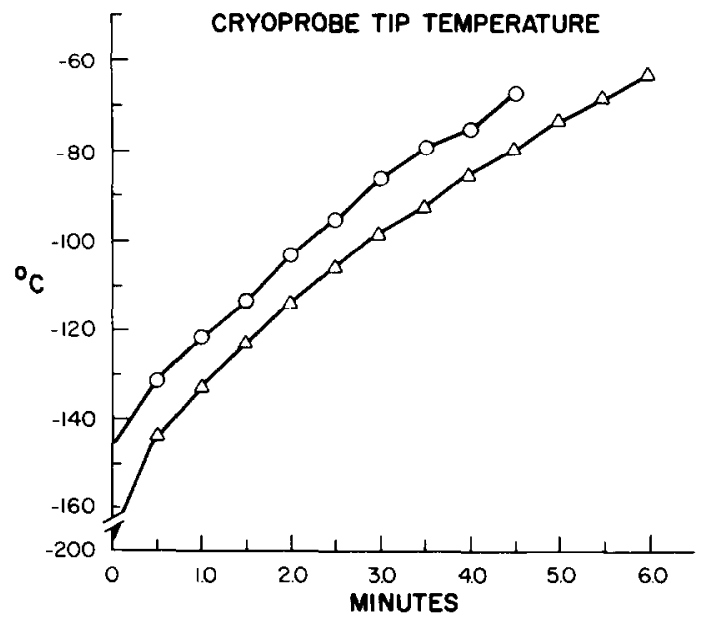

Fig. 2. Cryoprobe tip temperature recovery. Cryoprobe tip temperature was measured with a thermocouple embedded in the rod $1 \mathrm{~mm}$ from the tip. For each use, the copper portion of the probe was immersed in liquid nitrogen $\left(\mathrm{LN}_{2}\right)\left(-196^{\circ} \mathrm{C}\right)$ until temperature equilibration, then removed and applied. $0 \mathrm{~min}$, time of application; $(\mathrm{O})$ mean values from 5 apical applications; $(\Delta)$ probe removed from $\mathrm{LN}_{2}$ and left at room temperature. about $3 \mathrm{~min}$ and the cryoprobe was returned to the liquid nitrogen during this time. Survival time for these animals was 3-75 days.

Cochleas were fixed by perfusion with cold $2.5 \%$ glutaraldehyde and $2.0 \%$ paraformaldehyde in $0.1 \mathrm{M}$ phosphate buffer ( $\mathrm{pH} 7.4$ ), dissected in alcohol, and mounted as surface preparations for examination with differential interference-contrast (DIC) microscopy. The organ of Corti was evaluated from apex to base, the presence or absence of sensory cells recorded, and cytocochleograms made. Cells replaced by phalangeal scars or for which cell bodies could not be observed were recorded as absent. Further lesion characterization was confined to noting general condition of stereocilia, particularly as compared with control tissue. For cochleas with survival times of more than 30 days after cryoprobe application, the presence or absence of myelinated nerve fibers (MNFs) in the spiral osseous lamina was noted but quantitative evaluation was not possible under the preparation conditions used.

\section{Results}

\section{Single cryoprobe application}

In preliminary studies, single cryoprobe applications lasted $30 \mathrm{~s}$ and survival time was $30 \mathrm{~min}$ to 8 weeks. Apical damage to IHCs and OHCs in these animals was largely limited to stereocilia clumping and disarray for up to $30-40 \%$ of the distance from the apex. Application time was then increased on subsequent animals and survival time extended to a minimum of 2 days to allow for tissue healing and scar formation.

Table I summarizes the type and extent of lesions for cochleas damaged by single apical cryoprobe applications of $2.5,3.0$ and $3.5 \mathrm{~min}$. These 12 cochleas (of 20 examined) are representative of the range of damage for their respective groups. In most instances, there was a complete absence of the organ of Corti and/or a loss, indicated by scarring, of at least $75 \%$ of sensory cells for various distances from the apex. Nine of the 20 cochleas had such major damage for at least $20 \%$ of the distance from the apex (two examples are shown in Fig. 3A,B); one cochlea for 15\%; and 5 had major damage interrupted by a region of much less damage (Fig. 3C). Im- 
TABLE I

EXTENT OF HAIR CELL DAMAGE AFTER SINGLE APICAL CRYOPROBE APPLICATION

Twelve representative ears of 20 examined, after single cryoprobe applications of $2.5,3.0$, and 3.5 min. Survival refers to time between probe application and being killed. Upper line for each cochlea shows damage to inner hair cells (IHCs); lower line, damage to the 3 rows of outer hair cells (OHCs) combined. (草) Absence of sensory cells (includes scarring and absence of organ of Corti); (国) more than $75 \%$ of sensory cells replaced by scarring; ( $(B)$ up to $75 \%$ of sensory cells replaced by scarring; ( $\square$ ) stereocilia disarray and/or occasional scarring only.

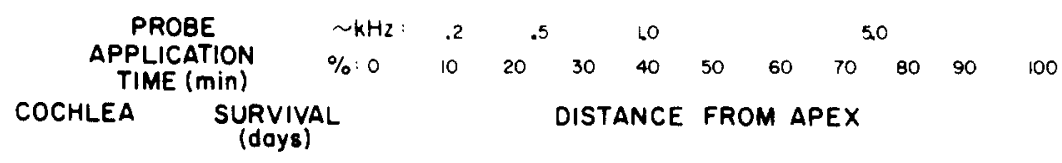
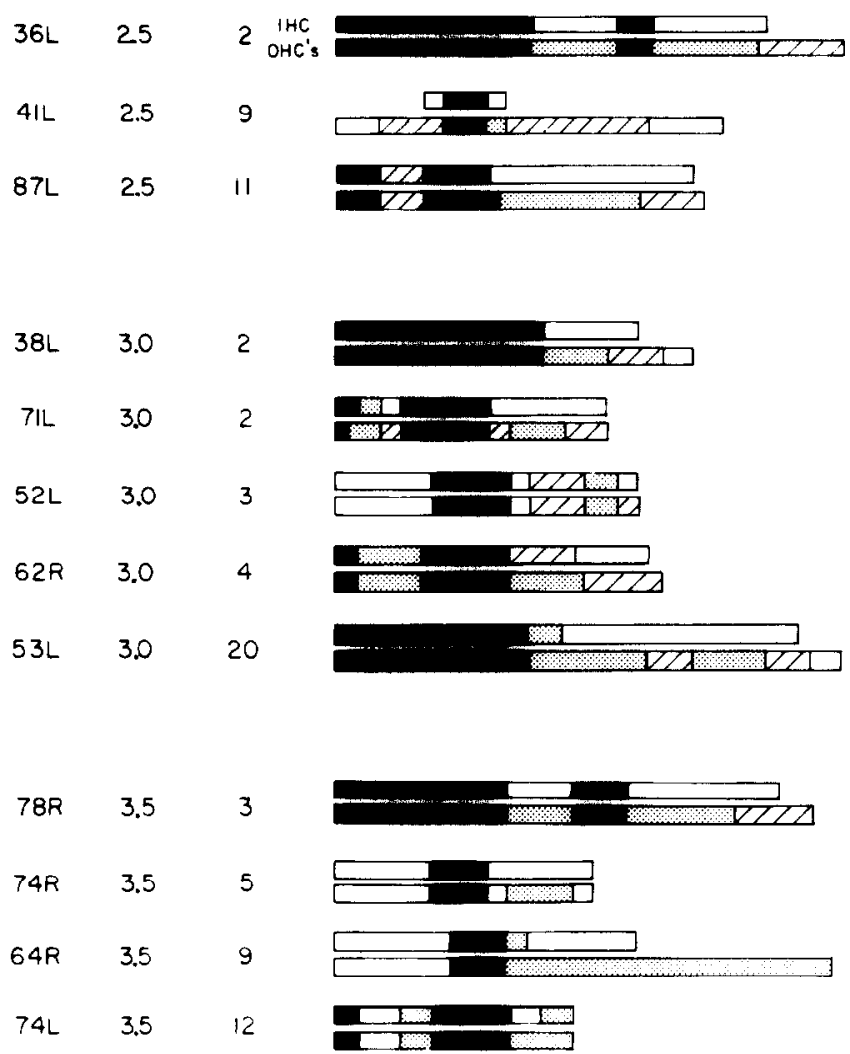

mediately basal to this major lesion area, there was frequently additional extensive scar formation but with less than $75 \%$ of the cells damaged (particularly in OHCs). Stereocilia disruption or occasional scarring comprised other regions of less damage. In 5 cochleas the lesions were judged unsatisfactory because damage was limited to stereccilia disruption and occasional scarring or because major damage occurred chiefly beyond $20 \%$ of the distance from the apex, while the apical region sustained less damage. In all but 4 of the 20 cochleas, moderate to heavy scarring or hair cell loss did not extend beyond the estimated frequency region above $5 \mathrm{kHz}$.

\section{Double cryoprobe application}

Four cochleas were exposed to two successive 2 or $3 \mathrm{~min}$ cryoprobe applications, at approximately the same apical site. Since their lesion areas were quite varied in extent and type of damage, two 

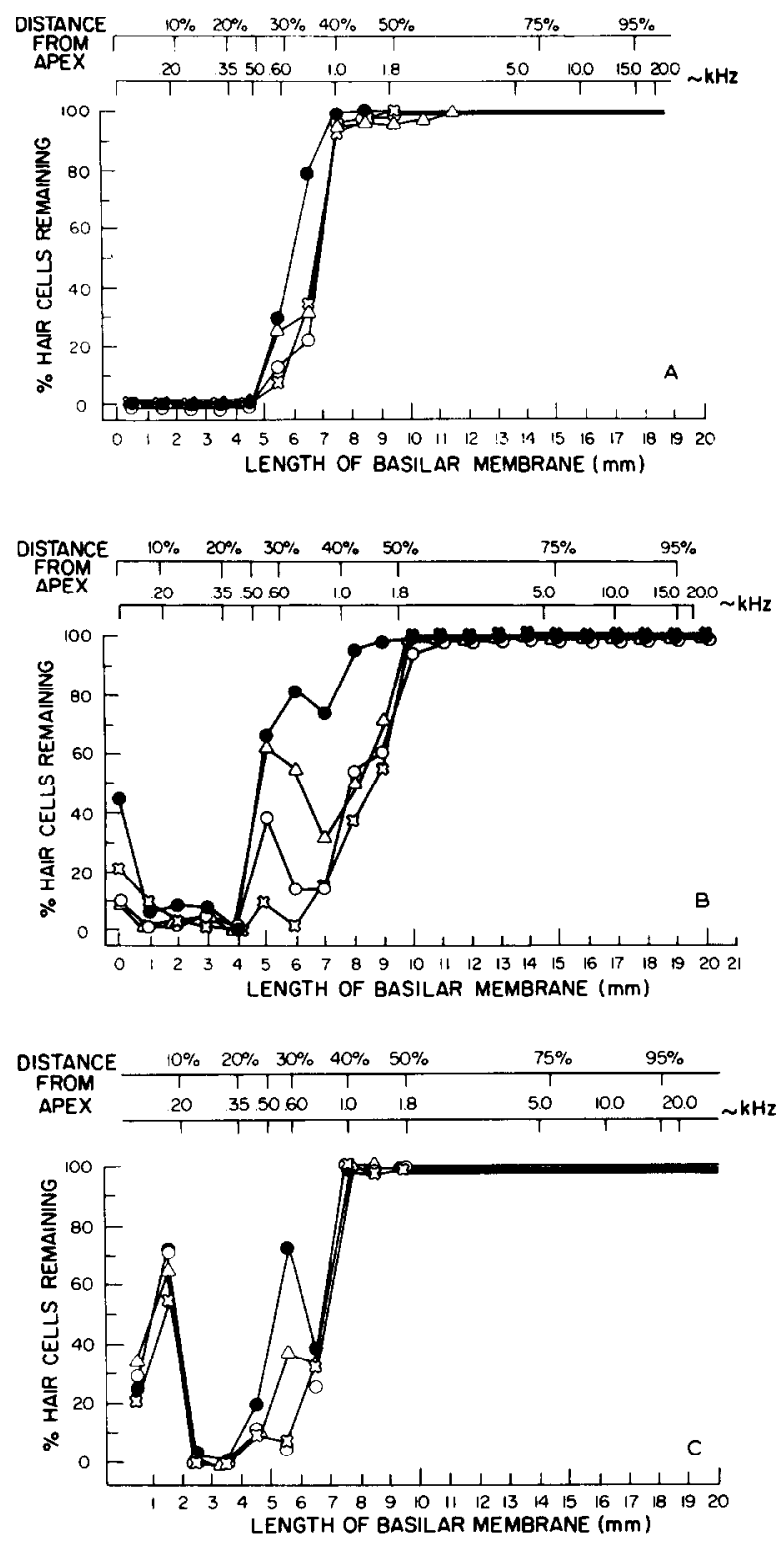

Fig. 3. Cytocochleograms of 3 cochleas after single cryoprobe application. (๑) Inner hair cells (IHC); (乏S) first row outer hair cclls $\left(\mathrm{OHC}_{1}\right)$; (O) $\mathrm{OHC}_{2} ;(\Delta) \mathrm{OHC}_{3}$. (A) Cochlea 52R. Cryoprobe applied to apex for $3 \mathrm{~min}$; survival time, 3 days. Apical exposure to the cryoprobe frequently results in complete loss of IHCs and OHCs and the usual configuration of the organ of Corti cannot be recognized. The region of transition from complete loss to normal-looking cells is quite narrow in this cochlea. More typically, it is broader, as in B. (B) Cochlea 62R. Cryoprobe applied to apex for $3 \mathrm{~min}$; survival time, 4 days. Outer hair cells are more susceptible to injury than are IHCs, and damage to them usually continues further basally than for IHCs. First row OHCs are generally most susceptible; second row, less so; third row, least. (C) Cochlea extending to around $70 \%$ of the distance from the apex, this exposure time was judged unsuitable for producing discrete apical lesions.

Two successive probe applications of $1.5 \mathrm{~min}$ each $(2 \times 1.5 \mathrm{~min})$ at about the same apical site were made on 13 cochleas from 8 animals (Table II). An interval of about $3 \mathrm{~min}$ between the applications was selected to allow the tissue to rewarm (Zacarian, 1977). The probe was immersed in liquid nitrogen during this time. In 9 of the 13 cochleas, major IHC damage consisted of complete absence of the organ of Corti and high loss of IHCs below $30 \%$ of the distance from the apex. Similar heavy damage to the corresponding OHCs occurred below $40 \%$, corresponding to estimated basilar membrane frequency tuning below $1 \mathrm{kHz}$ (Fig. 4A). In the remaining 4 ears, damage to both IHCs and OHCs was much more extensive, going beyond $60 \%$ of the distance from the apex.

Survival times after probe application were extended beyond 30 days for 1 animal and beyond 60 days for 4 others (total of 8 cochleas). Four of these cochleas exhibited damage patterns similar to those of cochleas with shorter survival times (less than 12 days), while in the remaining 4 cochleas damage had spread well beyond $60 \%$ of the distance from the apex.

\section{Lesion morphology}

All cochleas (except one in the single probe application group) exhibited at least one apical lesion area characterized by complete absence of sensory cells and supporting cells, in which the usual configuration of the organ of Corti could not be recognized. The absence of major damage to the one cochlea may have been due to poor probe tip contact with the wall of the cochlea. Immediately basal to the main lesion area there was always a region, of varying length, where sensory cell rows could be distinguished but also characterized by stereocilia disarray and missing hair cells indicated by scar formation. Damage to IHCs here was usually confined to stereocilia clumping and disarray and occasional missing

74L. Cryoprobe applied to apex for $3.5 \mathrm{~min}$; survival time, 12 days. In about $30 \%$ of the cochleas examined, major damage in the apex was interrupted by a region of less damage around $10 \%$ of the distance from the apex. 
cells. OHC damage, however, was much more severe and typically continued further basally than for IHCs. First row OHCs were most susceptible; second row, less so; and third row OHCs, least susceptible (Fig. 3). Further basally, OHC damage tapered off to occasional scars, followed by regular, even rows of OHCs with normal stereocilia configuration.

MNFs in the osseous spiral lamina were examined in the 8 cochleas with a survival time of more than 30 days. No MNFs were observed in areas where the organ of Corti was absent; in basal regions with normal hair cells, MNF condi- tion appeared comparable to control tissue. Nerve fiber population was reduced in transition regions between these two extremes.

Both single and double cryoprobe exposures can produce very discrete apical lesions (Figs. 3A, 4A). However, as illustrated in Table II, the pattern of severe apical damage after $2 \times 1.5 \mathrm{~min}$ probe applications is more consistent and repeatable than in single exposure cochleas in Table I.

Figs. $3 \mathrm{~A}$ and $4 \mathrm{~A}$ show a complete loss of both inner and outer apical hair cells and an abrupt transition area of moderate cell loss and stereocilia disarray. Sensory cells in the remaining organ

\section{TABLE II}

\section{EXTENT OF HAIR CELL DAMAGE AFTER TWO APICAL CRYOPROBE APPLICATIONS OF 1.5 MIN EACH}

Upper line for each cochlea shows damage to inner hair cells (IHC); lower line, damage to the 3 rows of outer hair cells (OHCs) combined. Survival refers to time between probe applications and being killed. There was a 3 min interval between the two 1.5 min applications. For symbols see Table I.

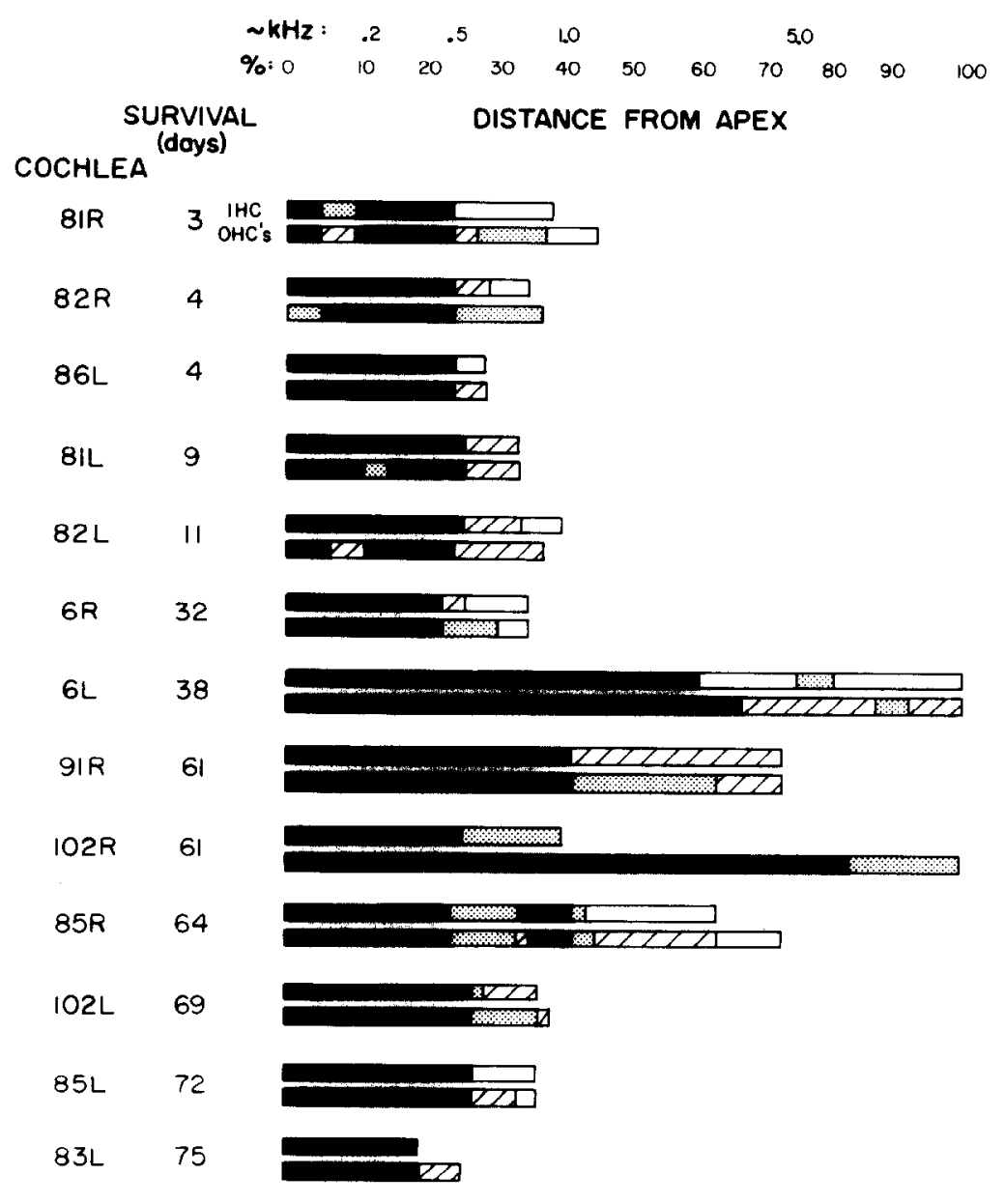



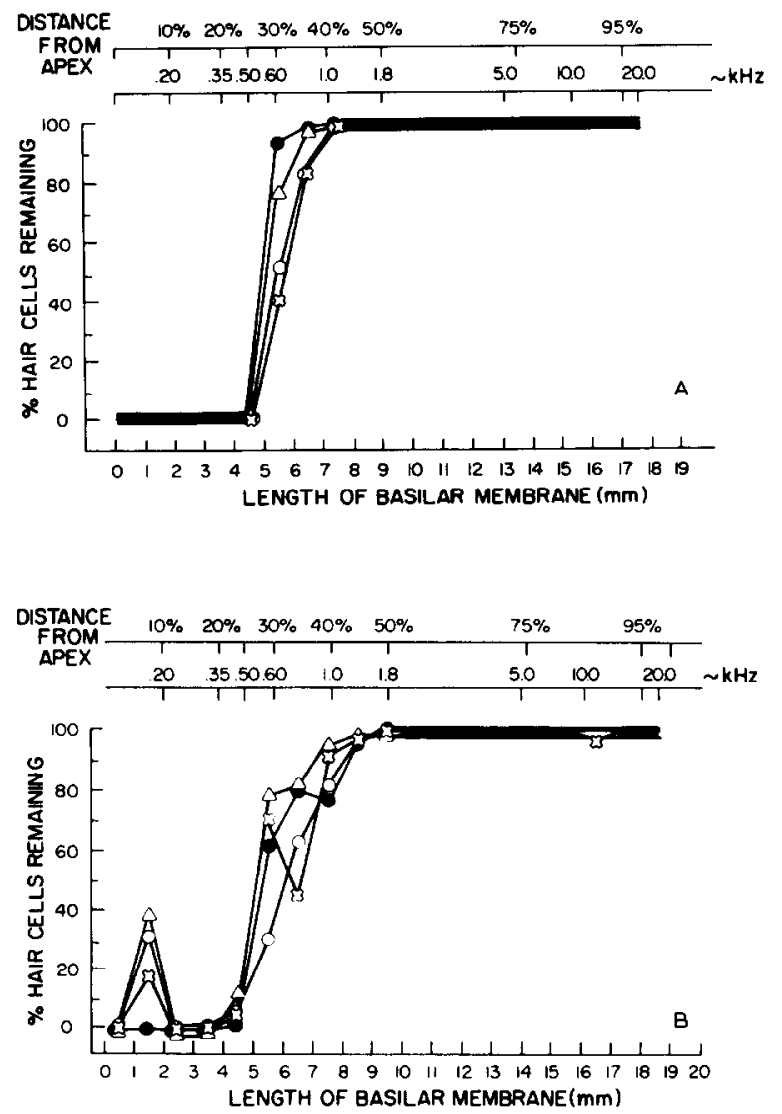

Fig. 4. Cytocochleograms of 2 cochleas after two $1.5 \mathrm{~min}$ apical cryoprobe applications, with an interval of $3 \mathrm{~min}$ between applications. (๑) $\mathrm{IHC}_{(\mathbb{3})} \mathrm{OHC}_{1} ;(\mathrm{O}) \mathrm{OHC}_{2} ;(\Delta) \mathrm{OHC}_{3}$. (A) Cochlea 85L, survival time, 72 days. Complete loss of apical organ of Corti, with abrupt transition region. (B) Cochlea $82 \mathrm{~L}$; survival time, 11 days.

of Corti are in regular even rows, with the usual stereocilia pattern. More typically, though, the transition area is broader, as in Figs. 3B,C and 4B. A region of less damage around $10 \%$ of the distance from the apex is also sometimes present (Figs. 3C and 4B, and Tables I and II).

Survival time for animals of both single and double exposure groups ranged from 2 to 75 days, with most being 12 days or less.

It is not clear from the data that longer survival time noticeably affects the extent of the lesion. For the 8 cochleas with a lesion-to-fixation time of 30 days or more (Table II), 4 showed notable damage beyond $60 \%$ of the distance from the apex. In the 3 animals which were lesioned in both ears $(2 \times 1.5 \mathrm{~min})$ and were allowed to survive beyond 30 days ( 1 animal) and 60 days ( 2 animals), all showed extensive damage in 1 ear only. The opposite ears exhibited apical lesions similar to those of the remaining 8 cochleas in that group.

\section{Discussion}

For the purpose of this study an ideal apical lesion was defined as a total loss of sensory cells from the most apical end of the cochlea to a point approximately $30-40 \%$ of the distance along the basilar membrane. A sharp transition region between missing hair cells and a normal-looking organ of Corti was also desirable. Of the several protocols investigated, a double application of the cryoprobe for $1.5 \mathrm{~min}$ with an interval of $3 \mathrm{~min}$ between resulted in the most successful lesion.

Single probe applications of $2.5-3.5 \mathrm{~min}$ also resulted in appropriate lesions but greater variability was noted in the overall extent of the damaged area and in the presence of islands of remaining (albeit damaged) hair cells.

The reasons for the observed variability and differential effect of multiple vs. single probe applications is a matter of conjecture since so many factors may be involved. The temperature recovery profile of the cryoprobe is consistent across applications but the degree of contact efficiency between the probe and the bony wall of the cochlea could be variable. It is possible that for multiple comparatively short time applications of the probe, the cooling of the cochlea is more localized and efficient because the probe temperature is on the lower portion of the probe-temperature recovery function. Also, evidence from other cryolesion studies in other tissues indicates that the thawing period of the freeze-thaw cycle is particularly damaging to biological tissue (Zacarian, 1977, 1980).

The results of the current study are consistent with those of Kuylenstierna and Lundquist (1982) and Suzuki and Hunter-Duvar (1982) who demonstrated that cryoprobe-induced lesions can be produced in the guinea pig and chinchilla, respectively. Kuylenstierna and Lundquist's (1982) light microscopic evaluation did not include a detailed assessment of stereocilia condition but a similar 
spread of damage as the current study seemed to occur with a single application of a commercial cryoprobe for approximately $30 \mathrm{~s}$. However, the extent of collapsed organ of Corti was much more limited. Suzuki and Hunter-Duvar evaluated cochlear morphology using scanning electron microscopy. This is undoubtedly a more sensitive measure of the spread of damage away from the lesioned area. Their cryolesion protocol was three 30-s applications (to slightly different locations of the apex) of a liquid nitrogen cooled metal probe which was similar in concept to the current study. Although percentage or millimeter measurements of the length of organ of Corti damage were not given, it appears that variability similar to the current study was noted; for 3 of 11 cochleas, the region of damage extended into the basal turn (in the current study this occurred for 4 of 13 cochleas when a second application was used; see Table II). The observation that inner hair cells are more resistant to damage and that the first row of outer hair cells most susceptible is confirmed in the current work.

Suzuki and Hunter-Duvar also reported that longer survival time (4 weeks) did not appreciably affect the extent of damage. However, 2 of 7 apically lesioned cochleas with survival times of 1-4 wk exhibited severe damage (by their rating scale) extending into the basal turn. Kuylenstierna and Lundquist (1982) found the healing process to be complete in guinea pig 6 days after lesioning, so survival times were not longer than this is their study. In the present investigation, auditory threshold changes after probe application were not followed electrophysiologically, hence any progression of the lesion with time cannot be demonstrated in these cochleas. However, the damage displayed in Table $I$ appears to be unrelated to survival time, and for the 3 animals in which both ears were lesioned $(2 \times 1.5 \mathrm{~min})$ and survival time was more than 30 days, all had extensive damage to one cochlea while damage to the other was confined to the apical $35-40 \%$. In an independent study (Smith et al., 1986a,b), 2 behaviorally trained animals with survival times of more than 70 days showed that post-lesion thresholds stabilized in about 10 days, and subsequent cytocochleograms showed apical lesions to about $25 \%$ and $35 \%$, respectively, and normal cell populations below the lesion. Such results suggest that lesion variability for the same probe application duration may be due more to differences in technique (e.g., probe placement and contact) and to slight physiological and anatomical differences between animals than to survival time alone.

The present study ideally required eradication of all apical sensory cells. Such loss of cells was achieved by Kuylenstierna and Lundquist (1982) in the guinea pig cochlea with cryounit temperatures of -40 and $-50^{\circ} \mathrm{C}$ (probe tip temperatures of around -30 to $-40^{\circ} \mathrm{C}$ ) and total cooling times of $30-40 \mathrm{~s}$. The completely disorganized area was rather small (1-1.5 $\mathrm{mm}$ in the probe tip region), but other types of severe cell damage continued much further basally. Cryounit temperatures of $-10,-20$, and $-30^{\circ} \mathrm{C}$ produced less damage. Also, the use of young animals and the slightly narrower and more free-standing apex in the guinea pig, compared to adult chinchilla, may permit greater cochlear cooling and cell injury. Damage rated 'severe' by Suzuki and HunterDuvar (1982) after three 30-s apical applications included considerable cell loss and cellular and stereocilia disruption, but a complete absence of sensory cells was not reported. Our single $30-\mathrm{s}$ applications were also ineffective in producing cell loss to the desired extent. Although the commercial cryosurgical unit is capable of holding a more constant temperature, the recorded temperatures of our probe tip and that used by Suzuki and Hunter-Duvar (1982) were well below those of the commercial instrument used in the Kuylenstierna and Lundquist $(1982,1983)$ study. Longer cold exposure time, therefore, seems an important factor in achieving a complete and extensive absence of organ of Corti. Two (or possibly more) applications with an appropriate re-warming interval may serve to limit the extent of the lesion area while increasing cell damage within it.

The apical lesions created by a simple cryoprobe in the current investigation are consistent enough to make this method a useful technique for the production of a new model to study cochlear function. Although complete morphological evaluation of the 'normal' basal region of the apically lesioned cochlea by transmission electron microscopy will be needed, the model appears well suited for the study of the behavioral and electro- 
physiological changes that have occurred. Such studies should reveal important, new information on low frequency encoding. This technique has been successfully used in behaviorally trained chinchillas, inducing threshold shifts with a $20 \mathrm{~dB}$ increase in frequencies below $1 \mathrm{kHz}$ (Smith et al., 1986a,b).

\section{Acknowledgements}

The authors thank Dr. Ivan Hunter-Duvar and Dr. Peter Thorne for their valuable suggestions regarding cryotechniques and morphological evaluation, respectively. This project was supported by Public Health Service NINCDS program project grant NS05785, and research grants 2 RO1 NS 15107 and 5 RO1 NS 11731.

\section{References}

Eldredge, D.H., Miller, J.D. and Bohne, B.A. (1981) A frequency-position map for the chinchilla cochlea. J. Acoust. Soc. Am. 69, 1091-1095.

Engström, H. and Ades, M.W. (1960) Effect of high-intensity noise on inner ear sensory epithelia. Acta Oto-Laryngol. Suppl. 158, 219-229.

Kuylensticrna, R. and Lundquist, P.-G. (1982) Experiments on cochlear cryosurgery in the guinea pig: Light and surface microscopy. Acta Oto-Laryngol. 94, 317-328.

Kuylenstierna, R. and Lundquist, P.-G. (1983) Experiments on cochlear cryosurgery in the guinea pig: Correlation of intracochlear temperature and morphology. Acta OtoLaryngol. Suppl. 393, 6-13.

Lundquist, P.-G., Igarashi, M., Wersäll, J., Alford, B.R. and
Wright, W.K. (1972) Effects of cryosurgery on vestibular sensory epithelia in the guinea pig. Arch. Otolaryngol. 95, 530-542.

Lundquist, P.-G., Schindler, R.A. and Stahle, J. (1978) Ultrasonic irradiation of guinea pig labyrinth. Acta Oto-Laryngol. $85,85-95$.

Morrison, M.D. and Lundquist, P.-G. (1974a) Intralabyrinthine temperature changes during cryosurgery in the guinea pig. Arch. Otolaryngol. 100, 220-225.

Morrison, M.D. and Lundquist, P.-G. (1974b) Labyrinthine morphology and temperature in cryosurgery (guinea pig). Acta Oto-Laryngol. 77, 261-273.

Smith, D.W., Brown, J.N., Moody, D.B., Stebbins, W.C. and Nuttall, A.L. (1986a) The effects of cryoprobe-induced apical lesions on behavioral auditory thresholds in the chinchilla. Abstr. 9th Midwinter Res. Meet. Assoc. Res. Otolaryngol., Feb. 1986, Clearwater Beach, FL.

Smith, D.W., Brown, J.N., Moody, D.B., Stebbins, W.C. and Nuttall, A.L. (1986b) Cryoprobe-induced apical lesions in the chinchilla. II. Effects on bchavioral auditory thresholds. Hear. Res. 26, 311-317.

Stahle, J., Hogberg, L. and Engström, B. (1972) The laser as a tool in inner ear surgery. Acta Oto-Laryngol. 73, 27-37.

Suzuki, M. and Hunter-Duvar, I.M. (1982) Cochlear effects of cryosurgery. Scan. Elec. Microsc. III, 1295-1300.

Winther, F.O. (1970) X-ray irradiation of the inner ear of the guinea pig. Thesis Universitetsforlaget. Oslo.

Ylikoski, J. (1974) Correlative studies on the cochlear pathology and hearing loss in guinea pigs after intoxication with ototoxic antibiotics. Acta Oto-Laryngol. Suppl. 326, $1-62$.

Zacarian, S.A. (1977) Cryolesion and the pathogenesis of cryonecrosis. In: Cryosurgical Advances in Dermatology and Tumors of the Head and Neck, pp. 9-14. Editor: S.A. Zacarian. C.C. Thomas Press, Springfield, IL.

Zacarian, S.A. (1980) Benign and malignant cutaneous lesions. In: Handbook of Cryosurgery, pp. 109-129. Editor: R.J. Ablin. Marcel Dekker, New York. 